\title{
EVENTOS NOTIFICADOS EM UMA UTI DE UM HOSPITAL PRIVADO, CERTIFICADO DO VALE DO PARAÍBA - SÃO PAULO, BRASIL.
}

\author{
Nilda da Silva Carvalho 1 \\ Jusleine Lima 1 \\ Claudia Lysia de Oliveira Araújo 2
}

\section{RESUMO}

Aenfermagem deve promover à prevenção de riscos e agravos à saúde dos pacientes, deve documentar todas as suas ações e intercorrências que aparecerem, pois essas informações poderão servir como fonte de conhecimento da história dos pacientes e investigação dos fatores que ocasionaram os eventos inesperados. São considerados eventos adversos lesões não intencionais que acabam resultando em incapacidade temporária ou permanente, tempo prolongado de internação ou até mesmo o óbito em consequência do cuidado prestado. 0 objetivo deste é identificar os eventos notificados de janeiro a julho de 2014 no hospital estudado. Trata-se de uma pesquisa, descritiva, quantitativa e exploratória, observou que dentre os pacientes internados os eventos adversos não infecciosos foram classificados como graves e ocorreram entre três e sete dias de internação relacionados à sonda enteral e os infecciosos corresponderam à pneumonia associada à ventilação mecânica.

Descritores: Acreditação, Assistência em enfermagem, Controle de Qualidade, Avaliação de Desempenho Profissional, Gestão de qualidade.

\section{ABSTRACT}

Nursing should promote the prevention of risks and health risks to patients, should document all your actions and events that appear, because this information may serve as a source of knowledge of the history of the patients and investigation of the factors that led to unexpected events. Adverse events are considered unintentional injuries that end up resulting in temporary or permanent disability, prolonged hospitalization or even death as a result of the care provided. The purpose of this is to identify the events reported from January to July 2014 in the hospital. This is a research, descriptive, exploratory and quantitative noted that among the patients admitted to the non-infectious adverse events were classified as serious and occurred between three and seven days of hospitalization related to enteral feeding and infectious corresponded to ventilator-associated pneumonia mechanical.

Keywords: Accreditation Assistance Nursing, Quality Control, Evaluation of Professional Performance, Quality Management.

1.Aluna de Graduação em Enfermagem das Faculdades Integradas Teresa D’Àvila - Fatea, Lorena, São Paulo, Brasil.

2. Professora Doutora em Enfermagem das Faculdades Integradas Teresa D’Àvila - Fatea, Lorena, São Paulo, Brasil 


\section{Introdução}

Atualmente hospitais e instituições optam pela busca constante na melhoria da qualidade do atendimento com o comprometimento de atender as necessidades de clientes internos e externos para aumentar o nível de satisfação dos mesmos quanto à segurança e a assistência prestada. Em 1999, foi criada no Brasil a Organização Nacional de Acreditação (ONA), sendo a mesma não governamental, sem fins lucrativos, caracterizada como pessoa jurídica de direito privado e com abrangência de atuação nacional. Seu objetivo geral é promover a certificação da qualidade dos serviços de saúde, onde se permite aprimorar a contínua atenção, garantindo qualidade na assistência em todas as organizações e instituições prestadoras de serviços de saúde do país, com a parceria com o Ministério da Saúde (1).

Em 1989 a Organização Mundial da Saúde (OMS), inseriu a acreditação hospitalar como elemento estratégico para desenvolver a qualidade da prestação dos serviços em instituições da América Latina, sendo assim a enfermagem se depara com a necessidade de aprimorar a qualidade do cuidado para garantir segurança aos pacientes, pois os mesmos se encontram cada vez mais bem informados sobres seus direitos como cidadão (2).

A acreditação é uma palavra originária do inglês e significa dar crédito, reconhecer, certificar, portanto é um método que as organizações hospitalares utilizam para avaliar o ambiente, os materiais, prontuários e 0 trabalho da equipe de enfermagem dentro das instituições, certificando-as e permitindo que as mesmas continuem funcionando. Esse processo está elevando e aperfeiçoando a cada dia mais a qualidade do atendimento, promovendo segurança, impedindo que intercorrências e falhas que podem ser prevenidas aconteçam, superando assim as expectativas dos clientes (3).

Para o desenvolvimento do processo de acreditação, não é avaliado apenas um serviço ou um departamento isoladamente, mas é considerado o hospital ou instituição como um todo, pois se entende que as estruturas e os processos são interligados, onde o funcionamento de um componente acaba interferindo no outro e também no resultado final. A acreditação hospitalar funciona como educação permanente ao pessoal de serviço e de seus líderes, utilizando uma metodologia de consenso, racionalização e ordenamento dos hospitais (4).

A segurança do paciente hospitalizado é a principal característica que demonstra a qualidade do serviço prestado pela equipe de enfermagem. A diminuição dos riscos são desafios constantes da equipe de saúde e sendo assim requer profissionais devidamente treinados e qualificados para boa qualidade dos cuidados prestados (5).

A qualidade do atendimento sempre esteve presente desde os primórdios da humanidade, quando o cuidado era realizado por pessoas de acordo com o conhecimento e experiência de vida que possuíam e que julgavam correto e melhor possível para beneficiar o outro. Com o avançar dos anos foram surgindo profissionais da saúde e instituições hospitalares e com eles reflexões sobre a qualidade dos serviços oferecidos. A enfermagem entra nesse contexto com importante papel, pois é ela que se articula com toda a equipe multiprofissional e com o universo do paciente procurando incentivar a todos para alcançar um bem comum (6).

Existem organizações acreditadoras que fazem a avaliação de hospitais a fim de certificar a qualidade dos serviços prestados pela equipe e analisar se estes cuidados estão sendo desempenhados de acordo com exigências estabelecidas por metodologias que vem sendo aplicadas, no entanto esta prática não é obrigatória e sim voluntária (7).

As instituições prestadoras de serviços de grande importância social, que possuem alta complexidade e peculiaridades como hospitais e que se atenta para o aperfeiçoamento da qualidade de atendimento, possuem diferencial específico e se tornam vencedoras, pois criam alternativas e avançam com maior rapidez identificando e melhorando suas fragilidades, criando mecanismos inovadores a cada dia (8).

São considerados eventos adversos, lesões não intencionais que acabam resultando em incapacidade temporária ou permanente, tempo prolongado de internação ou até mesmo o óbito, em consequência do cuidado prestado. Com o 
avanço das tecnologias desenvolveu-se novas especialidades em saúde evoluindo assim os cuidados prestados pela equipe (9).

Novos equipamentos foram sendo criados para auxiliar e facilitar a realização de procedimentos mais complexos para a garantia da vida. A complexidade de tais aparelhos é mais vista dentro de setores que possuem pacientes mais graves como pronto socorro e unidade de terapia intensiva. Como são máquinas, requer

manutenção apropriada, verificação do funcionamento, limpeza adequada constantemente, portanto 0 gerenciamento de risco contínuo dos aparelhos eletrônicos é de extrema importância (10).

O atendimento com qualidade, principalmente em instituições hospitalares tem como objetivo principal, manter os pacientes livres de riscos e danos trazendo-Ihes segurança e satisfação. As ocorrências indesejáveis consideradas como eventos adversos podem ser prevenidas quando seguidos exigências de programas e organizações de acreditação e certificação. Os indicadores de resultados são importantes para o gerenciamento se tornando possível avaliar a qualidade da assistência. Vários autores, ao investigarem os eventos adversos e as consequências para os pacientes, destacaram alguns erros com sondas, drenos e cateteres, erros de medicação, queda de pacientes, extubações não programadas, úlceras por pressão e infecções adquiridas em internações. 0 programa de acreditação hospitalar consiste em avaliar processos assistenciais através de normas padronizadas, previamente estabelecidas objetivando melhorar a qualidade da assistência e oferecer maior segurança aos pacientes. Esta certificação vem ganhando força nos últimos anos dentro das instituições hospitalares (11).

A enfermagem deve promover a prevenção de riscos e agravos à saúde dos pacientes, mas também deve documentar todas as suas ações e intercorrências que aparecerem, pois informações precisas devidamente registradas poderão servir como fonte de conhecimento da história dos pacientes, investigação dos fatores que ocasionaram eventos inesperados e tem valor como documento legal. Os registros podem servir como instrumentos para examinar as condições da assistência prestada pela enfermagem, portanto é imprescindível o preenchimento correto, com letra legível, domínio da língua e com todas as informações necessárias, pois a indiferença e a desatenção podem esconder pontos preciosos do estado de saúde dos pacientes (12).

Os eventos adversos no cuidado com o cliente podem surgir a todo instante, desde administração de medicamento pela via incorreta até uma equipe não qualificada, estrutura física desapropriada, entre muitos outros. São situações que expõe os clientes a riscos que até então não tinham, principalmente clientes de UTI, pois estão mais debilitados e vulneráveis. Nesse setor a avaliação dos eventos adversos é fundamental, pois existem clientes em estado grave, equipamentos tecnológicos e um grande número de profissionais, sendo necessárias atenção e

intervenções adequadas para a prevenção de erros, bem como a organização do processo de trabalho, checagem dos equipamentos e acompanhamento da evolução dos pacientes (13).

Ainda é pouco divulgada no Brasil a identificação, classificação e análise dos Eventos Adversos a Medicamentos (EAMs) que ocorrem em instituições hospitalares. São poucas as ações governamentais a esse respeito, pensando na segurança dos pacientes. Em 2001 o Ministério da Saúde, através da Agência Nacional de Vigilância Sanitária (ANVISA), criou uma rede de hospitais sentinela os quais estavam preparados para notificar eventos adversos, porém ainda não existe banco de dados a nível nacional que possa receber e analisar essas ocorrências e também são poucos os hospitais que possuem um sistema formal de cadastramento dessas notificações e analise dos erros (1).

Notificar algo é comunicar a alguém um fato ocorrido. Nos hospitais a notificação é um instrumento muito importante para o gerenciamento de enfermagem, para informar e alertar as diferentes modalidades de profissionais atuantes quanto a riscos, possibilitando a todos a refletir sobre novas condutas que promovam a prevenção e segurança dos pacientes (14). 
A escolha do tema teve como principal motivação o fato do grande aumento de Eventos Adversos que ocorrem dentro das instituições e isto nos levou a pesquisar o quanto a Acreditação pode ajudar a mudar tal situação e melhorar 0 atendimento, proporcionando total segurança aos usuários do serviço oferecido dano ao mesmo uma referência que garante assistência com qualidade e traça normas e regras visando prever erros, principalmente com os pacientes de Unidade de Terapia Intensiva que estão mais debilitados necessitando de maiores cuidados.

A notificação dos Eventos Adversos é muito importante para que o gerenciamento de enfermagem possa informar e se atentar para as diferentes modalidades de profissionais atuantes quanto aos riscos.

\section{Objetivos}

Conhecer os eventos notificados em uma UTI de um Hospital privado, certificado em uma cidade do Vale do Paraíba, São Paulo, Brasil; caracterizar quanto ao tipo de instituição, número de leitos e política de atendimento e identificar os tipos e números de ocorrências de eventos notificados de Janeiro a Julho/2014.

\section{Métodos}

Trata-se de uma pesquisa descritiva com abordagem quantitativa e exploratória em um hospital privado, certificado em uma cidade do Vale do Paraíba, São Paulo, Brasil.

Os critérios de inclusão foram os registros, sistema e prontuários da UTI no período de Janeiro a Julho de 2014. Os critérios de exclusão foram as demais internações em outras unidades.

Uma vez aprovado o projeto pelo Comitê de Ética da Fatea sob n ${ }^{0} 359.687$, foi agendado com a Coordenadora do hospital a data para a coleta dos dados. Foi garantido a todos o seu anonimato; a garantia de não haver quaisquer sansões ou prejuízos pela não participação ou pela desistência, a qualquer momento; o direito de resposta às dúvidas; a inexistência de qualquer ônus financeiro ao participante.

Para a coleta de dados foi utilizado o relatório composto por registros, sistemas e prontuários dos pacientes internados na UTI no período de Janeiro a Julho de 2014.

Os dados foram analisados e descritos a seguir.

\section{Resultados e Discussões}

A instituição foi inaugurada em 1980 como gastroclinica. Em 2002 inaugurou a UTI. Instalada em uma área com mais de $10.000 \mathrm{~m}^{2}$, é a primeira do Vale do Paraíba a ter sua qualidade certificada pela ONA, além de ser a única com a Acreditação por Excelência, uma garantia de atendimento seguro e eficiente. Recebeu a acreditação ONA II em 2006 passando para ONA III em 2012. Também é o único hospital da região associado à Associação Nacional dos Hospitais Privados (ANAHP), entidade que reúne os hospitais privados brasileiros com os melhores padrões de qualidade.

Parceiro dos principais convênios médicos, o hospital conta com instalações e equipamentos modernos, PA Clínico e Ortopédico nas $24 \mathrm{~h}$ e estrutura completa para a realização de cirurgias de todos os portes, em diversas especialidades. Possui 64 leitos, sendo 16 leitos de UTI's, 1.353 médicos cadastrados no corpo clínico aberto, 367 empregos diretos e 600 indiretos, 69.693 atendimento no pronto socorro por

ano, 19.356 consultas ambulatoriais no centro médico por ano e 5.125 cirurgias realizadas por ano.

Conta com alta tecnologia e infraestrutura completa para a realização de cirurgias de todos os portes e diversas 
especialidades, como neurologia, cardiologia, oncologia, gastroenterologia, entre outras com quatro salas de cirurgias e área de recuperação pós-anestésica. Oferece excelência na realização de procedimentos cardíacos, diagnóstico e tratamento de doença coronária, cerebrovasculares, cardiovasculares, patologias adquiridas e congênitas com atendimento $24 \mathrm{hs}$, equipamentos de hemodinâmica que produz imagens integradas, tridimensionais e tomográficas e equipe multidisciplinar preparada para recepcionar e cuidar dos pacientes.

O centro médico possui atendimento clínico com tratamentos em diversas especialidades como alergologia, angiologia, cardiologia, arritmologia, coloproctologia, dermatologia oncológica, gastroenterologia, hepatologia, nutrição, oncologia clínica, ortopedia, clínica geral e cirurgias como vascular, buco maxilo facial, aparelho digestivo, cardíaca e neurocirurgia.

Trabalha com rigorosos critérios internacionais de controle de qualidade e de humanização, onde conta com uma UTI adequada ao Sistema Brasileiro de Indicadores - Qualidade em Terapia Intensiva (QUATI). Dentre os 16 leitos de UTI, dois leitos são de isolamento com Sistema Fluxolaminar Linear, que filtra 0 ar 16 vezes por hora, e tem controle de pressão ambiente com Sistema de Pressão Positiva e Negativa, baseado nos critérios de Serviço de Controle de Infecção Hospitalar (SCIH), que reduz o risco de contágio de doenças infectocontagiosas. Há um moderno sistema de monitorização para todos os leitos, possibilitando que os pacientes sejam rigorosamente acompanhados pela equipe médica e de enfermagem. O paciente é monitorado desde a chegada ao hospital até a alta, por um cirurgião, um médico especialista e uma equipe de enfermagem treinada para observar e agir prontamente no caso de qualquer intercorrência.

No período de estudo houve 627 (100\%) internações na UTI, sendo que dez internações $(1,6 \%)$ foram internações remanescentes do período anterior.

Segundo estudo e pesquisa em uma UTI adulta do HC-UNICAMP, durante o período de seis meses estiveram internados 422 pacientes. (15).

Na UTI do Hospital Nossa Senhora da Conceição em Porto Alegre-RS, foi realizado uma pesquisa quanto às internações durante o período de seis meses e registraram 607 internações (16).

Quanto ao fluxo de pacientes atendidos, houve uma taxa de ocupação de 93\% com duração média de cinco dias de internação, com desfecho total de 574 (91,55\%) altas, 37 (5,9\%) óbitos e $16(2,55 \%)$ não informados.

A pesquisa feita no Hospital Regional de Guarabira- Paraíba mostra o tempo de permanência do paciente na UTI em média de cinco dias (17).

Segundo pesquisa desenvolvida uma UTI adulta do HC-UNICAMP, o tempo médio de permanência do paciente é de um a seis dias. Entretanto, com 0 aperfeiçoamento de conhecimentos e o desenvolvimento das novas tecnologias, 0 paciente gravemente enfermo é mantido por um período prolongado nessas unidades, ocasionando alto custo financeiro, distúrbios morais e psicológicos para todos os envolvidos (15).

O tempo médio de permanência dos pacientes internados na UTI adulto de um hospital escola no Paraná, segundo pesquisa foi de sete dias (18).

Dentre as principais características Clinicas de Internação na UTI no período de estudo, observou-se que 339 (54\%) eram do sexo masculino, $288(46 \%)$ eram do sexo feminino, dando destaque então para os homens, sendo a média de idade dos pacientes 55 anos.

No Hospital Regional de Guarabira - Paraíba, à distribuição da amostra segundo o sexo, considerando 140 (100\%) pacientes estudados, observou-se que $71(51 \%)$ eram do sexo feminino e $69(49 \%)$ do sexo masculino, observando-se 
o destaque maior para o sexo feminino. A idade mínima observada foi de 18 anos, enquanto a máxima foi de 94 anos. A média de idade foi de 65 anos (17).

Na UTI de uma Instituição Pública em Fortaleza-CE, do total de 384 (100\%) pacientes pesquisados, 227 (59\%) correspondem ao sexo masculino e 157(41\%) ao sexo feminino, prevalecendo o número maior para o sexo masculino (19).

No estudo realizado na UTI de um hospital escola no Paraná, do total das 1305 (100\%) internações durante um período de seis meses, 796 (61\%) eram do sexo masculino e 509 (39\%) do sexo feminino, mostrando que nesse período houve predomínio do sexo masculino em relação ao feminino (18).

Ainda dentro das características em nossa pesquisa, observou-se que 13

pacientes (2\%) são <18 anos de idade, 194 pacientes (31\%) estão entre 18-44 anos, 238 pacientes (38\%) entre 45-64 anos, 113 pacientes (18\%) entre 65-80 anos e 69 pacientes (11\%) são >80 anos de idade.

Em estudos feitos em uma UTI adulta do HC-UNICAMP, o número de internações foi de 422 pacientes, porém foram inclusos no estudo 401 (100\%) pacientes sendo 239 (59,6\%) homens e 162 (40,4\%) mulheres. A média de idade encontrada foi de 54 anos. Este estudo apresentou também que 90 (22,4\%) dos pacientes apresentaram menos que 40 anos de idade, 155 (38,6\%) entre 40 e 60 anos, e 156 (39\%) estavam acima de 60 anos (15).

Na UTI de uma instituição pública de Fortaleza-CE observou-se há predominância de idosos na faixa etária de 61 a 70 anos, que somados aos demais com 71 anos ou mais, representaram a maioria (19).

Quanto aos motivos de admissões na UTI, as patologias clínicas atingiram 345 internações (55\%), obtendo um predomínio em relação às cirurgias eletivas com 251 internações (40\%) e cirurgias de urgência/emergência com 31 internações.

Segundo a pesquisa realizada em uma UTI de um hospital Universitário em João Pessoa - PB em um período de seis meses no ano de 2012 houve um total de 126 internações, porém devido a dados incompletos 26 pacientes foram excluídos da pesquisa, sendo a mesma realizada com apenas 102 pacientes, sendo que os principais motivos que levaram os pacientes a serem internados na UTI foram nove (8,82\%) Diabetes Mellitus, nove (8,82\%) Cardiopatias, oito $(7,84 \%)$ Insuficiência Respiratória Aguda - IRA e sete (6,86\%) Acidente Vascular Encefálico - AVE (20) .

Outra pesquisa registra 357 (100\%) como principais motivos de internações na UTI sendo, 286 (80\%) casos clínicos e $71(20 \%)$ casos cirúrgicos. E de acordo com os prontuários analisados destes pacientes, 144 (40,3\%) já haviam passado por cirurgias anteriores (21).

Conforme mostra a pesquisa realizada em uma UTI de um hospital universitário dentre os 104 pacientes envolvidos na pesquisa os motivos que levaram à internação identificaram que as principais causas foram às doenças infecciosas em $38(28 \%)$ pacientes, as neurológicas em 34 (25\%), seguida pelas doenças metabólicas em $17(12 \%)$ e respiratórias em 17 (12\%). Além desses, outros nove (7\%) internaram por problemas cardiovasculares, nove (7\%) por lesões e/ou doenças traumáticas, seis (4\%) por neoplasias e sete $(5 \%)$ por outras causas.

Deve-se levar em consideração que alguns destes pacientes se internaram com mais de um motivo (22).

De acordo com a procedência esta pesquisa constatou que as maiorias das internações tiveram sua origem da unidade de emergência atingindo 250 internações (39,9\%), em seguida observa-se 213 (34\%) internações cirúrgicas, 63 internações (10\%) da enfermaria, 63 internações (10\%) da sala de hemodinâmica, 19 internações (3\%) transferência de 
outra unidade hospitalar, 15 (2,5\%) internações de outra UTI, duas (0,3\%) internações procedentes de home-care e duas $(0,3 \%)$ internações de procedência não especificada, conforme a tabela 1.

Tabela 1 - Distribuição das internações ( $\mathrm{N}=627$ ) dos pacientes internados no período de Janeiro a Julho, em UTI de um hospital privado, certificado do Vale do Paraíba. 2014.

\begin{tabular}{|l|c|c|}
\hline Unidade & N & $\%$ \\
\hline Unidade de Emergência & 250 & $39,9 \%$ \\
Centro Cirúrgico & 213 & $34 \%$ \\
Enfermaria & 63 & $10 \%$ \\
Sala de Hemodinâmica & 63 & $10 \%$ \\
unidade hospitalar & 19 & $3 \%$ \\
$\begin{array}{l}\text { Unidade de Terapia } \\
\text { Intensiva - UTI }\end{array}$ & 15 & $2,5 \%$ \\
Home-Care & 2 & $0,3 \%$ \\
Não especificado & 2 & $0,3 \%$ \\
\hline
\end{tabular}

Na UTI de uma instituição pública de Fortaleza-CE, a procedência dos 384 (100\%) pacientes internados foi de 249 $(64,8 \%)$ da unidade de emergência, confirmando então que essa UTI cumpre o seu maior propósito, que é absorver a grande demanda de emergência (19).

Em uma UTI de um hospital universitário, quanto à procedência, observou-se predominância de 41 (39,4\%) pacientes que eram do PA, seguido por 19 (18,3\%) pacientes procedentes da clínica médica e 18 (17,3\%) pacientes da sala de recuperação anestésica. Os demais 20 (19,2\%) pacientes provieram de outras unidades da instituição. Além disso, seis $(5,8 \%)$ pacientes eram provenientes de outras instituições do munic

Do total de 627 (100\%) internações, observou-se como principais medidas de suporte á internação neste período $100(16 \%)$ medidas, sendo $50(8 \%)$ na utilização de ventilação mecânica, 31(5\%) para suporte renal e $19(3 \%)$ para aminas.

Sobre a caracterização dos principais eventos adversos e sentinela não infecciosos verificou-se a existência de 30 $(4,8 \%)$ dos pacientes internados, sendo um total de $45(100 \%)$ eventos registrados no período. Dos $45(100 \%)$ eventos registrados, 17 eventos (38\%) foram classificados como grave, 13 eventos (29\%) como moderada, seis eventos (13\%) como leves e nove eventos (20\%) não foram informadas.

Referente à distribuição dos eventos adversos e sentinelas invasivos constatou-se que 14 (31\%) eventos correspondiam à sonda enteral, nove $(20 \%)$ eventos às Úlceras por Pressão (UPP), quatro (9\%) eventos tubo endotraqueal, quatro ( $9 \%$ ) eventos por cateter venoso central, três (7\%) eventos com flebite por punção de veia periférica, três $(7 \%)$ eventos relacionados à barotrauma, dois $(4,5 \%)$ eventos por cateter venoso - PICC, dois $(4,5 \%)$ eventos relacionados à cânula de TQT, dois (4\%) eventos relacionados a erros e dois (4\%) eventos relacionados á complicações 
com hemotransfusão e acidente de punção venosa, conforme apresenta a tabela 2.

Tabela 2 - Eventos adversos e sentinelas invasivos relacionados à internação dos pacientes no período de Janeiro a Julho, em UTI de um hospital privado, certificado do Vale do Paraíba. 2014.

\begin{tabular}{|l|c|c|}
\hline Unidade & Eventos & $\%$ \\
\hline Sonda Enteral & 14 & $31 \%$ \\
Úlcera Por Pressão & 9 & $20 \%$ \\
Tubo Endotraqueal & 4 & $9 \%$ \\
Cateter Venoso Central & 4 & $9 \%$ \\
Flebite - punção de veia periférica & 3 & $7 \%$ \\
Barotrauma & 3 & $7 \%$ \\
Acidente de punção venosa & 2 & $4 \%$ \\
Cateter Venoso - PICC & 2 & $4 \%$ \\
Cânula de Traqueostomia & 2 & $4 \%$ \\
$\begin{array}{l}\text { Erros e complicações relacionados } \\
\text { à Hemotransfusão }\end{array}$ & 2 & \\
\hline
\end{tabular}

As UPP foram registradas nove (100\%) sendo distribuídas em seis (67\%) em região sacra, um (11\%) em trocânter e outros dois $(22 \%)$ em outras regiões.

Quanto à classificação da lesão de UPP, um (11\%) evento foi encontrado em estágio I e oito (89\%) eventos foram encontrados em estágio II.

De acordo com as ocorrências registradas da lesão de UPP no total de nove $(100 \%)$ eventos, quanto período de internação, observou-se que três (34\%) eventos ocorreram com pacientes que ficaram internados por um período de três a sete dias, dois (22\%) eventos entre oito e 14 dias, um (11\%) eventos entre 15 e 30 dias, dois (22\%) eventos superiores a 30 dias e um (11\%) evento com período de até dois dias de internação.

Para intubação, houve um total de quatro eventos no período do estudo sendo dois (50\%) eventos por desposicionamento ou remoção do tubo pelo paciente e dois $(50 \%)$ eventos por desposicionamento ou remoção do tubo acidentalmente correspondendo a um total de quatro (100\%) eventos de gravidade.

Os eventos adversos com Cânula de Traqueostomia (TQT) totalizaram dois (100\%) no período, sendo um (50\%) evento por desposicionamento ou remoção acidental e um (50\%) evento devido à obstrução.

Segundo a gravidade do evento um (50\%) evento foi classificado como grave e um (50\%) evento classificado como leve sem maiores repercussões. 
Referente aos principais eventos relacionados ao suporte ventilatório, do total de 627 (100\%) pacientes internados no período com os mais variados diagnósticos, constatou-se que $63(10 \%)$ dos pacientes estavam em ventilação mecânica em duração média de 13 dias.

Em relação ao barotrauma houve um total de três (100\%) eventos adversos no período sendo todos relacionados a pneumotórax e classificados como grave.

Quanto à caracterização dos principais eventos adversos relacionados a infecções, encontrou-se a pneumonia associada à ventilação mecânica totalizando um evento no período e infecções do trato urinário totalizando três eventos. Foram utilizados um total de 1706 cateteres/dia correspondendo a uma taxa de utilização de 54\%; e por fim infecções de corrente sanguínea contabilizando total de dois eventos com comprovação laboratorial.

De acordo com a pesquisa feita em uma UTI de um Hospital Universitário as infecções hospitalares incidiram em $52(50 \%)$ da população estudada. As infecções respiratórias foram as que mais apareceram com 59 (57\%), seguidas das infecções da corrente sanguínea com 24 (23\%) e infecções urinárias com 12 (12\%). Sepse e choque séptico, também, apareceram entre as morbidades mais frequentemente adquiridas, durante a internação (22).

Para os procedimentos e medidas de suporte invasivo durante a Internação na UTI dos pacientes egressos da unidade, obteve-se um total de 611 saídas incluindo altas e óbitos.

Quanto ao destino dentro do período de estudo saíram 611 (100\%) do hospital sendo que, 235 (38,5\%) dos internados foram encaminhados a Enfermaria, 241 (39,5\%) foram para outra Unidade de Cuidados Intermediários (UCl) do hospital, $11(2 \%)$ foram transferidos para outro hospital, $37(6 \%)$ tiveram óbito e $84(14 \%)$ voltaram para suas residências.

A pesquisa do Hospital Regional de Guarabira - Paraíba mostra o desfecho das 140 (100\%) internações na UTI considerando três opções: alta da UTI, transferência para outro hospital ou óbito e durante o período estudado, observouse os seguintes resultados: 67 (47,85\%) pacientes foram a óbito, 70 (50\%) pacientes receberam alta da UTI, enquanto que apenas três $(2,15 \%)$ pacientes foram transferidos para outro hospital (17).

Na UTI de uma Instituição Pública de Fortaleza-CE, quanto ao encaminhamento dos 384 pacientes pesquisados, observou-se que 286 (74,5\%) foram transferidos para a clinica cardiológica, $63(16,4 \%)$ evoluíram para o óbito, 31 (8,1\%) foram transferidos para outro hospital e quatro $(1,0 \%)$ tiveram alta hospitalar (19).

Em uma pesquisa na UTI de um Hospital Universitário os dados mostraram que dos 104 pacientes envolvidos na pesquisa, 52 (50\%) pacientes foram a óbito e 52 (50\%) tiveram alta (22).

\section{Considerações Finais}

Observamos que existem programas de controle de registro de eventos adversos dentro das instituições e que se mantidos atualizados os dados de uma forma fidedigna por toda a equipe é possível fazer uma avaliação das ocorrências registradas e trabalhar com toda a equipe a fim de melhorar a qualidade do atendimento ao paciente e familiar por meio de treinamentos, cursos de atualizações e até mesmo realizando melhorias de equipamentos.

Este trabalho contribuirá para que as instituições interessadas em apresentar boa qualidade de atendimento aos seus pacientes e familiares tenham base segura de que vale a pena investir na busca pela certificação a fim de melhorar a qualidade do serviço prestado, pois a instituição certificada reduz gastos desnecessários com processos reparadores de danos aos clientes por problemas que podem ser evitados seguindo normas e diretrizes da certificação. 


\section{Considerações Finais}

A Instituição em estudo por ser acreditada por excelência oferece garantia de atendimento seguro e eficiente aos clientes. Possui 64 leitos, sendo 16 leitos de U.T.I. onde conta com alta tecnologia e infraestura completa para a realização de cirurgia de todos os portes e diversas especialidades.

Durante o período da pesquisa observou-se que o maior número de pacientes atendidos foi do sexo masculino, classificados como idosos, com predomínio de internações por motivos clínicos, originando-se da Unidade de Emergência.

As principais medidas de suporte a internação foram ventilação mecânica.

Quanto à caracterização dos principais eventos adversos não infecciosos

observou-se que a maioria dos eventos foram classificados como grave e sua distribuição correspondendo a Sonda Enteral e UPP. Os registros de eventos ocorreram com maior frequência na primeira semana de internação. Quanto à administração de fármacos não houve nenhum erro no período.

Para os eventos adversos infecciosos observamos a pneumonia associado à ventilação mecânica como o evento mais registrado.

Quanto aos registros de óbitos observou-se que na unidade em estudo a taxa foi bem inferior aos demais locais de estudo e pesquisa.

\section{Referências}

1.Silva, C. S., et al. Opinião do enfermeiro sobre indicadores que avaliam a qualidade na assistência de enfermagem. Rev. Gaúcha de Enfermagem, v.30, n. 2, 2009.

2.Vituri, D. W., et al. Indicadores de qualidade como estratégia para melhoria da qualidade do cuidado em um hospital universitário. Rev. Ciência, Cuidado e saúde. v. 9 n. 4, 2010.

3.Feldman, L. B.; Gatto, M. A. F.; Cunha, I. C. K. I. O. História da evolução da qualidade hospitalar: dos padrões a acreditação. Acta Paul Enferm. 18(2): 213-9 2005.

4.Lima, S. B. S., Erdmann, A. L. A enfermagem no processo da acreditação hospitalar em um serviço de urgência e emergência. Acta. Paulista. Enferm. vol.19 no.3 São Paulo, julho/setembro. 2006.

5.Arboit, E. L. Eventos Adversos na Enfermagem relacionados à terapia medicamentosa. Jornal da Enfermagem UNIFRA. p.4 2012.

6.Labbadia, L. L., et al. O Processo de acreditação hospitalar e a participação da Enfermeira. Rev. Enfermagem UERJ. c. 12; p.83-87, 2004.

7.Filho, J. F. C., Montenegro, A. V., Barragão, S. R. M. S. Qualidade na Saúde a luz da acreditação hospitalar e sua interface com gestão de pessoas. Portal dos Psicólogos, Psicologia.pt. 2012.

8.Bonato, V. L. Gestão de qualidade em saúde: melhorando assistência ao cliente. O mundo da saúde. São Paulo, 35(5): 319-331 2011. 
9.Brito, MJ. M., Rocha, A. M.; Ferreira, V. R. Análise dos eventos adversos em uma unidade de terapia intensiva neonatal como ferramenta de gestão da qualidade da

assistência de enfermagem. Rev. Eletrônica quadrimestral de Enfermagem. n.17 Outubro, 2009.

10.Florence, G., Calil, S. J. Uma nova perspectiva no controle dos riscos da utilização de tecnologia médico-hospitalar. Rev. Tecnologia para a saúde, MultiCiência. outubro, 2005.

11.Nascimento, C. C. P., et al. Indicadores de resultados da Assistência: Análise dos eventos adversos durante a internação hospitalar. Rev. Lat-Am Enfermagem. 16 (4), julho-agosto, 2008.

12.Setz, V. G., D'innocenzo, M. Avaliação da qualidade dos registros de Enfermagem no prontuário por meio da auditoria. Rev. Acta Paul. Enferm. 22(3):313-7, 2009.

13.Beccaria, L. M.; et al. Eventos adversos na assistência de Enfermagem em uma Unidade de Terapia Intensiva. Rev. Bras. Ter. Intensiva. 21(3): 276-282, 2009.

14.Paiva, M. C. M. S., Paiva, S. A. R., Beerti, H. W. Eventos adversos: análise de um instrumento de notificação utilizado no gerenciamento de enfermagem. Rev. esc. enferm. USP. São Paulo, v.44, n.2 Jun, 2010.

15.Oliveira, A. B. F., et. al. Fatores associados à maior mortalidade e tempo de internação prolongado em uma unidade de terapia intensiva de adultos. Rev Bras Ter Intensiva. 2010; 22(3):250-256.

16.Moraes, R. S., Fonseca, J. M. L., Leoni, C. B. R. D. Mortalidade em UTI, Fatores Associados e Avaliação do Estado Funcional após a Alta Hospitalar. Revista Brasileira Terapia Intensiva. ano 2005.

17.Bezerra, G. K. A. Unidade de Terapia Intensiva - Perfil de Admissões: Hospital Regional de Guarabira, Paraíba, Brasil. Periódicos, UFPB, v. 16, n.4, 2012.

18.Costa, J. B.; Marcon, S. S.; Mombelli, M. A. Caracterização das Internações em uma Unidade de Terapia Intensiva UTI adulto de um hospital escola. Rev. Ciência, cuidado e saúde, v. 7, 2008.

19.Rocha, M. S.; et. al. Caracterização da população atendida em Unidade de Terapia Intensiva: Subsídio para a assistência. R Enferm UERJ, Rio de Janeiro, 2007 jul/set; 15(3):411-6.

20.França, C. D. M., Albuquerque, P. R., Santos, A. C. B. C. Perfil Epidemiológico da Unidade de Terapia Intensiva de um Hospital Universitário. InterScientia, João Pessoa, v.1, n.2, p. 72-82, maio/ago. 2013.

21.Garcia, T. P. R., et. al. Principais motivos de internação do paciente com Insuficiência Renal Aguda na Unidade de Terapia Intensiva. Rev. Ciência da Saúde - FAMERP, p. 146-50, julho/setembro 2005.

22.Favarin, S. S. Camponogara, S. Perfil dos pacientes internados na unidade de terapia intensiva adulto de um hospital universitário. Rev. UFSM v.2, n.2 2012. 